ARTICLE

https://doi.org/10.1038/s41467-019-08817-7

\title{
Photosynthetic apparatus of Rhodobacter sphaeroides exhibits prolonged charge storage
}

Sai Kishore Ravi (1) ${ }^{1}$, Piper Rawding ${ }^{2}$, Abdelnaby M. Elshahawy (1) ${ }^{1}$, Kevin Huang ${ }^{3}$, Wanxin Sun ${ }^{4}$, Fangfang Zhao ${ }^{4}$, John Wang ${ }^{1}$, Michael R. Jones ${ }^{5} \&$ Swee Ching Tan (1) ${ }^{1}$

Photosynthetic proteins have been extensively researched for solar energy harvesting. Though the light-harvesting and charge-separation functions of these proteins have been studied in depth, their potential as charge storage systems has not been investigated to the best of our knowledge. Here, we report prolonged storage of electrical charge in multilayers of photoproteins isolated from Rhodobacter sphaeroides. Direct evidence for charge build-up within protein multilayers upon photoexcitation and external injection is obtained by Kelvinprobe and scanning-capacitance microscopies. Use of these proteins is key to realizing a 'selfcharging biophotonic device' that not only harvests light and photo-generates charges but also stores them. In strong correlation with the microscopic evidence, the phenomenon of prolonged charge storage is also observed in primitive power cells constructed from the purple bacterial photoproteins. The proof-of-concept power cells generated a photovoltage as high as $0.45 \mathrm{~V}$, and stored charge effectively for tens of minutes with a capacitance ranging from 0.1 to $0.2 \mathrm{~F} \mathrm{~m}^{-2}$.

\footnotetext{
${ }^{1}$ Department of Materials Science and Engineering, National University of Singapore, 9 Engineering Drive 1, Singapore 117575, Singapore. ${ }^{2}$ Department of Materials Science and Engineering, University of Wisconsin-Madison, 1509 University Ave, Madison, WI 53706, USA. ${ }^{3}$ Department of Materials Science and Engineering, University of Illinois at Urbana-Champaign, 1304W Green St, Urbana, IL 61801, USA. ${ }^{4}$ Bruker Nano Surface Division, 11 Biopolis Way \#10-10, The Helios, Singapore 138667, Singapore. ${ }^{5}$ School of Biochemistry, University of Bristol, Biomedical Sciences Building, University Walk, Bristol BS8 1TD, UK. Correspondence and requests for materials should be addressed to S.C.T. (email: msetansc@nus.edu.sg)
} 
ighly quantum-efficient biophotonic processes that occur in natural photosynthetic proteins have a huge implication for future energy technologies. In a general sense, the term biophotonic process refers to a phenomenon related to the interaction of biological matter with photons ${ }^{1}$. Some of the most widespread and impactful examples are the photophysical and photochemical processes that take place during the initial steps of photosynthesis ${ }^{2-4}$. These involve the interaction of lightharvesting complexes with incoming photons to form pigment excited electronic states, ultrafast (picosecond time scale) energy transfer to efficiently move excited state energy to a reaction centre (RC) pigment-protein, and trapping of energy through photochemical charge separation ${ }^{2-4}$.

Besides being the core biophysical processes in nature, the processes of energy harvesting, conversion, and storage underlie many existing energy technologies ${ }^{5-7}$. In energy research, these three processes are usually realized separately through a modular device architecture ${ }^{8}$, with each requiring optimization and effective interfacing to achieve a high overall efficiency ${ }^{9}$. Typically, solar, thermal, chemical, or mechanical energy harvested through a variety of mechanisms is converted into electrical energy and, if not used directly, stored using a capacitor or a battery ${ }^{10,11}$. As there are losses involved at each stage, considerable efforts have gone into the development of hybrid systems where two or more of the three processes are combined. In some cases, energy harvesting and conversion systems might be one and the same, but devices typically have a distinct system performing the energy storage function. An emerging example of a hybrid system is the "self-charging" power cell or supercapacitor, the basic function of which is to harvest and convert ambient energy into electricity and continuously charge a battery or capacitor to ensure a sustainable supply of power ${ }^{12,13}$. The majority of these power cells are based on piezoelectric ${ }^{14,15}$ or triboelectric $^{16-18}$ nanogenerators that harvest ambient (bio) mechanical energy and store converted energy in supercapacitors or batteries. Fewer attempts have been made to develop selfcharging power cells that harvest light energy, these mainly involving the hybridization of dye-sensitized solar cells with supercapacitors ${ }^{19,20}$. Hybrid devices have also been described in which Li-ion batteries are photocharged by perovskite solar cells $^{21}$, and recently a hybrid self-charging power cell was reported that combined a photoelectrochemical cell with a redoxflow battery ${ }^{22}$. These hybrid power cells typically contain a lightharvesting/conversion system and an electrical energy storage system combined into a single-device architecture, sometimes with a common electrode.

On studying the light harvesting and charge transport properties of a purple bacterial photosynthetic protein, multilayers of the protein are able to store the photo-generated charges for a long time spanning tens of minutes. Investigating the origin of this phenomenon and demonstrating the implication this can have for energy research formed the basis of this work. Microscopic and macroscopic evidence of this phenomenon are presented using Kelvin-probe force microscopy (KPFM), scanning capacitance microscopy (SCM), photochronopotentiometry, cyclic voltammetry $(\mathrm{CV})$, and galvanostatic charge-discharge studies.

Exploiting the "prolonged charge storage" behavior, we construct photoprotein-based biophotonic power cells (BPCs, Fig. 1a) in which the light harvesting, energy conversion, and charge storage processes are integrated within a single, multifunctional material. This has been realized through the use of a pigment-protein complex from a natural bacterial photosystem that absorbs energy across the solar spectrum and transiently stores harvested energy through charge separation ${ }^{23-26}$. Such photosystems either in the form of isolated protein complexes or a

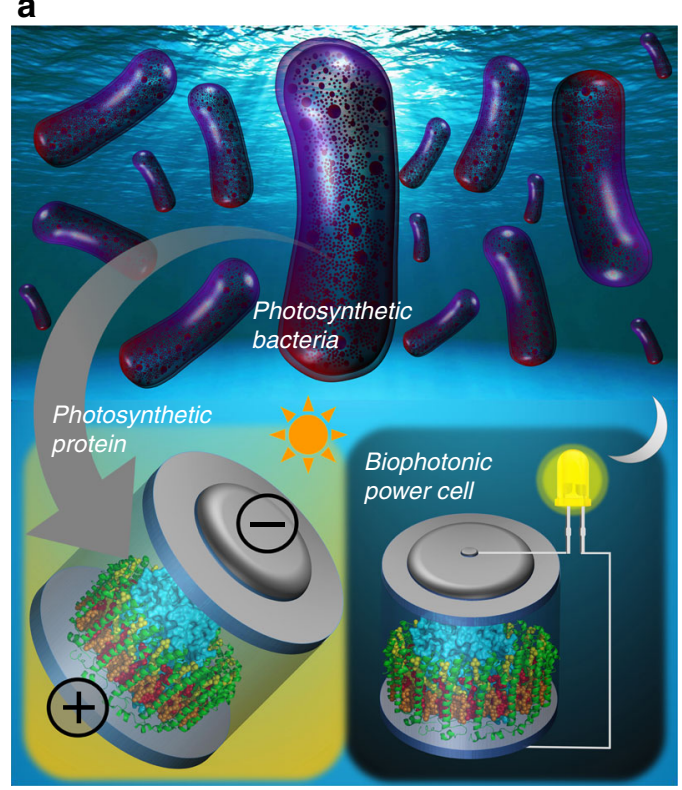

b



Fig. 1 Photosynthetic proteins, charge separation, and cell architecture. a Cartoon illustrating the concept of a biophotonic power cell based on natural photosynthetic components. b The RC-LH1 complex comprises a central reaction centre (RC) charge separation domain (cyan) surrounded by a cylindrical light harvesting ( $\mathrm{LH} 1)$ domain. c The RC can be isolated as a separate complex and is shown as a solid object with a transparent surface in the plane of the membrane to reveal the electron transfer cofactors. Photoexcitation of the $\mathrm{P}$ bacteriochlorophyll (BChl) pair initiates a four-step charge separation via a $\mathrm{BChl}$, bacteriopheophytin (BPhe) and ubiquinone (UQ) to reduce the $\mathrm{Q}_{B} \cup \mathrm{Q}$ on the opposite side of the photosynthetic membrane. d The LH1 cylinder can be isolated as a separate complex and is formed from 32 BChls (alternating red/orange) and 32 carotenoids (yellow) held in place by a protein scaffold (green). e Assembled cells comprised a multilayer of purified RC-LH1 proteins (green LH1 with cyan RC) sandwiched between n-Si and FTO-glass electrodes. $\mathbf{f}$ Under illumination a photovoltage is produced due to light-activated protein/electrode redox interactions, but current does not flow through the protein multilayer in the absence of mobile mediators 

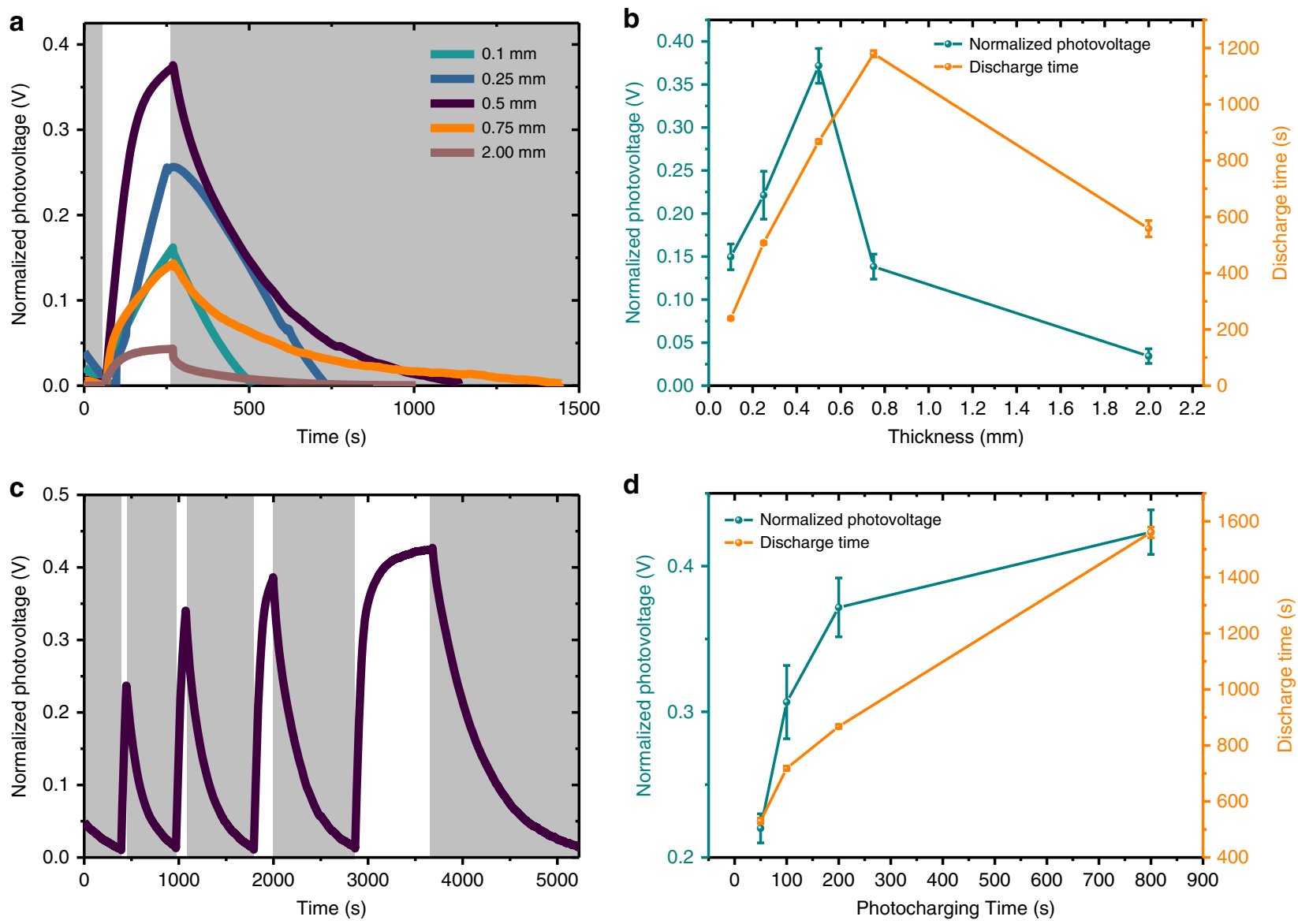

Fig. 2 Charging and discharging of biophotonic power cells. $\mathbf{a}, \mathbf{b}$ Effect of protein film thickness on photocharging and discharging in biophotonic power cell (BPC) with RC-LH1 (reaction centre-light harvesting 1 complex). c, d Effect of photocharging time on the photovoltage and discharge time in $500 \mu \mathrm{m}$ RCLH1 BPCs. In $\mathbf{a}$ and $\mathbf{c}$, gray = light-off and white = light-on. In $\mathbf{b}$ and $\mathbf{d}$, data points are the average values from three replicates with error bars representing a standard deviation

as whole bacterial cells are increasingly studied for biophotovoltaic and bioelectronic applications ${ }^{27-35}$. The particular photoprotein used, the so-called PufX-deficient RC-light harvesting 1 (RC-LH1) complex from the photosynthetic bacterium Rhodobacter (Rba.) sphaeroides (Fig. 1b), is an integral membrane pigment-protein made up from a central RC domain (Fig. 1b, c, cyan) surrounded by a cylindrical LH1 antenna complex (Fig. 1b, d) ${ }^{23,24,36,37}$. Following the absorption of solar energy by the bacteriochlorophylls (BChls) and carotenoids of the LH1 domain (Fig. 1d, and Supplementary Fig. 1), the key energy conversion event is a four-step electron transfer between a pair of $\mathrm{BChl}$ molecules $(\mathrm{P})$ at one end of the RC protein and a ubiquinone molecule $\left(\mathrm{Q}_{\mathrm{B}}\right)$ at the opposite end (Fig. 1c) ${ }^{25,26}$. This forms a charge-separated state $\left(\mathrm{P}^{+} \mathrm{Q}_{\mathrm{B}}{ }^{-}\right)$around $1 \mu \mathrm{s}$ after photoexcitation that is stable for a few seconds (Fig. 1c). To fabricate a simple device that generates and stores the photogenerated charges, films of concentrated photoprotein of varying thickness are sandwiched between a transparent fluorine-doped tin oxide (FTO) glass front-electrode and an n-doped silicon (n-Si) back electrode (Fig. 1e, f). We demonstrate that the resulting BPC carries out solar energy harvesting, energy conversion, and energy storage in a single, integrated architecture.

\section{Results}

Construction and photoresponse of a BPC. BPCs were assembled by drop casting a $20-1000 \mu \mathrm{L}$ aliquot of concentrated RC-
LH1 protein solution (see Materials and methods) into a well formed from one or more layers of plastic paraffin film (Parafilm M) adhered to an FTO-glass slide. This enabled formation of a protein multilayer of regular area and thicknesses between 0.1 and $2 \mathrm{~mm}$. After partially drying the protein under a vacuum a precleaned $n$-type silicon counter electrode was sandwiched with the protein-coated FTO electrode and the cell was sealed. The result was a densely packed protein multilayer formed in the absence of any additional electrolyte to act as a charge carrier. For reference, a single RC-LH1 complex has a maximum diameter of $\sim 13 \mathrm{~nm},{ }^{23}$ which means that a closely packed, $0.1 \mathrm{~mm}$-thick multilayer should be the equivalent of a minimum of $\sim 7700$ stacked protein monolayers, and $\sim 154,000$ monolayers for a $2 \mathrm{~mm}$ film. The absorbance characteristics of RC-LH1 complexes in solution are shown in Supplementary Fig. 1.

Five RC-LH1 BPCs of varying thickness were charged by exposure to 1 sun illumination for $200 \mathrm{~s}$ under open-circuit conditions. The photovoltage achieved increased with the thickness of the protein multilayer up to $500 \mu \mathrm{m}$, beyond which the maximum photovoltage dropped (Fig. 2a). In addition to the highest photovoltage under this standard illumination period ( $\approx 0.37 \mathrm{~V}$; Fig. $2 \mathrm{~b}$ ), the $500 \mu \mathrm{m}$ film exhibited the second longest dark discharge time (Fig. 2b). For these $500 \mu \mathrm{m}$ cells, the photovoltage increased as the photocharging time was increased (Fig. 2c, d), as did the dark discharge time. The longest discharge time obtained was $1560 \mathrm{~s}(26 \mathrm{~min})$ for a charging time of $800 \mathrm{~s}$ (Fig. 2d). 


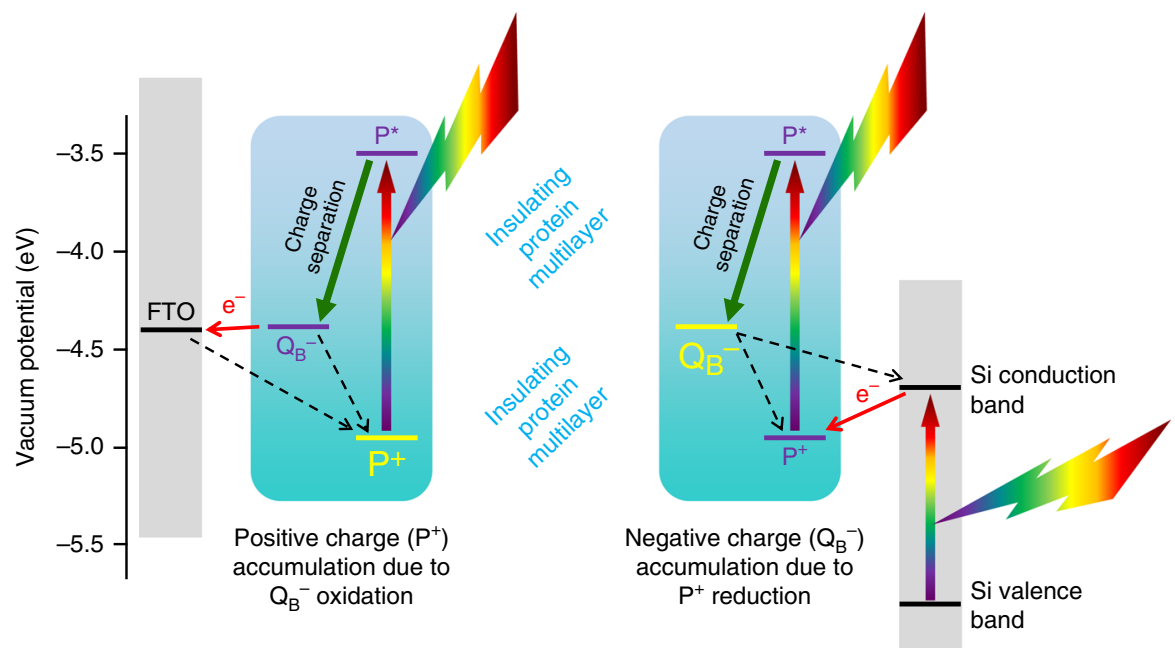

Fig. 3 Photoexcitation and electrochemical activity at the two electrodes. In cells with RC-LH1 proteins (i.e., reaction centre-light harvesting 1 complexes), in the photo-excited protein layers near the FTO (fluorine-doped tin oxide) electrode (left), trapped positive charges (initially $\mathrm{P}^{+}$) accumulate as $\mathrm{Q}_{\mathrm{B}}{ }^{-}$ formed by intra-RC charge separation (green arrow) donates electrons to the FTO (red arrow). In photo-excited protein layers near the n-Si electrode (right), trapped negative charges (initially $\mathrm{Q}_{B}{ }^{-}$) accumulate as $\mathrm{P}^{+}$formed by intra-RC charge separation (green arrow) is reduced by the photoactive $\mathrm{n}-\mathrm{Si}$ electrode (red arrow). In the absence of an electrolyte, these protein/electrode interactions cause the build-up of a potential difference over time. At either electrode, the process responsible for the generation of trapped positive or negative charges would be expected to be in competition with wasteful competing reactions (dashed black arrows)

Origin of the voltage build-up. Taking into account vacuum potentials, and a well-established understanding of the photochemistry of RC-LH1 complexes ${ }^{25,26}$, the observed photovoltage is attributed to net oxidation of RC-LH1 proteins at the FTO electrode (Fig. 3, left) and net reduction of RC-LH1 proteins at the $\mathrm{n}$-Si electrode (Fig. 3, right), producing trapped charges on opposite sides of the protein multilayer. The initiating event, in either case, is the photogeneration of the radical pair $\mathrm{P}^{+} \mathrm{Q}_{\mathrm{B}}{ }^{-}$ (Fig. 1c), which has a lifetime of $3-5 \mathrm{~s}$ in purified RC-LH1 complexes $^{38-40}$, and which relaxes by recombination at the $\mathrm{P}$ BChls. Net oxidation of protein at the FTO-glass electrode would be achieved if $\mathrm{Q}_{\mathrm{B}}{ }^{-}$in a suitably oriented $\mathrm{RC}-\mathrm{LH} 1$ protein is able to reduce the FTO more rapidly than either $\mathrm{P}^{+} \mathrm{Q}_{\mathrm{B}}{ }^{-}$recombination or reduction of $\mathrm{P}^{+}$by the FTO (Fig. 3, left). At the photoactive $\mathrm{n}$-Si electrode (Fig. 3, right), a net reduction of the adjacent protein would be possible if electrons from the conduction band of the $\mathrm{n}$-Si were able to reduce $\mathrm{P}^{+}$more rapidly than either $\mathrm{P}^{+} \mathrm{Q}_{\mathrm{B}}{ }^{-}$recombination or donation of an electron to the conduction band of the $\mathrm{n}-\mathrm{Si}$ by $\mathrm{Q}_{\mathrm{B}}{ }^{-}$.

The observed gradual build-up of the photovoltage (Fig. 2c) indicated that the density of trapped charges increased over several hundred seconds. This can be attributed to multiple oxidation or reduction events within individual complexes and/or propagation of trapped electrons or holes deeper into the multilayer through slow interprotein electron transfer. Regarding the former, it has been estimated from electrochemical titrations that an LH1 ring can store up to eight $\mathrm{BChl}$ cations without undergoing irreversible photo-oxidative damage ${ }^{41}$, and multiple negative charges can be accumulated on the quinone and bacteriochlorin cofactors of the $\mathrm{RC}$ when the rate of reduction of the photo-oxidized RC by an external donor exceeds the rate of charge recombination or quinone oxidation by an external acceptor $^{42,43}$. In addition, RCs contain a single dissociable $\mathrm{Q}_{\mathrm{B}}$ ubiquinone that can undergo double reduction, the second reduction being accompanied by double protonation, and preparations of RC-LH1 complexes typically contain 10-15 molecules of ubiquinone in the space between the $\mathrm{RC}$ and the surrounding LH1 ring that can exchange with the quinone at the
$\mathrm{Q}_{\mathrm{B}}$ site $^{38}$. As a result it is plausible that a single RC-LH1 complex could accumulate more than one positive or negative charge if adjacent to an electrode that can act as an acceptor/donor. Regarding charge propagation, as RCs undergo charge separation under illumination, each can be treated as an electric dipole. In an ideal scenario where all dipoles were aligned with positive ends facing one electrode and negative ends facing the other, charge storage in the device could be possible without any transport mechanism in the active layer. However, since such a device configuration with perfectly aligned dipoles is unrealistic for proteins assembled into a multilayer, charge storage in the device is likely to also rely on the transport of charges between complexes in those layers closest to the surface of either electrode. It is highly probable that interprotein interfaces in the device act as charge localization sites allowing charges to hop from one state to other before they reach the electrodes. Incoherent hopping of charge carriers from site to site is known to be a dominant transport mechanism in disordered systems and has also been studied previously in photosynthetic systems ${ }^{44-46}$. Such gradual penetration of long-lived trapped charges into the photoactive protein multilayer through electron hopping between adjacent proteins could also be a factor in the slow discharge of the photovoltage on terminating the illumination period. (Fig. 2d). Although not of the same nature as the capacitive origin of voltage build-up in dry protein multilayers in this device, trends of slow photoresponse have been previously observed in liquid biophotoelectrochemical cells despite the addition of electron transport mediators ${ }^{29,47-49}$, where slow rise and decay in photovoltage were the direct effect of slow electrolyte diffusion ${ }^{47}$.

It should be noted that while charge transport could play an important role in the build-up of a charge difference between electrodes, it is crucial that long-range transport from one electrode to the other is avoided as this would counteract the charge storage ability of the BPC. In an ideal dielectric capacitor, there will not be any charge transport through the device and the charge storage is achieved purely by the accumulation of charges at the electrode/dielectric interface. In the BPCs the protein layers in the center of the device therefore serve the function of an 

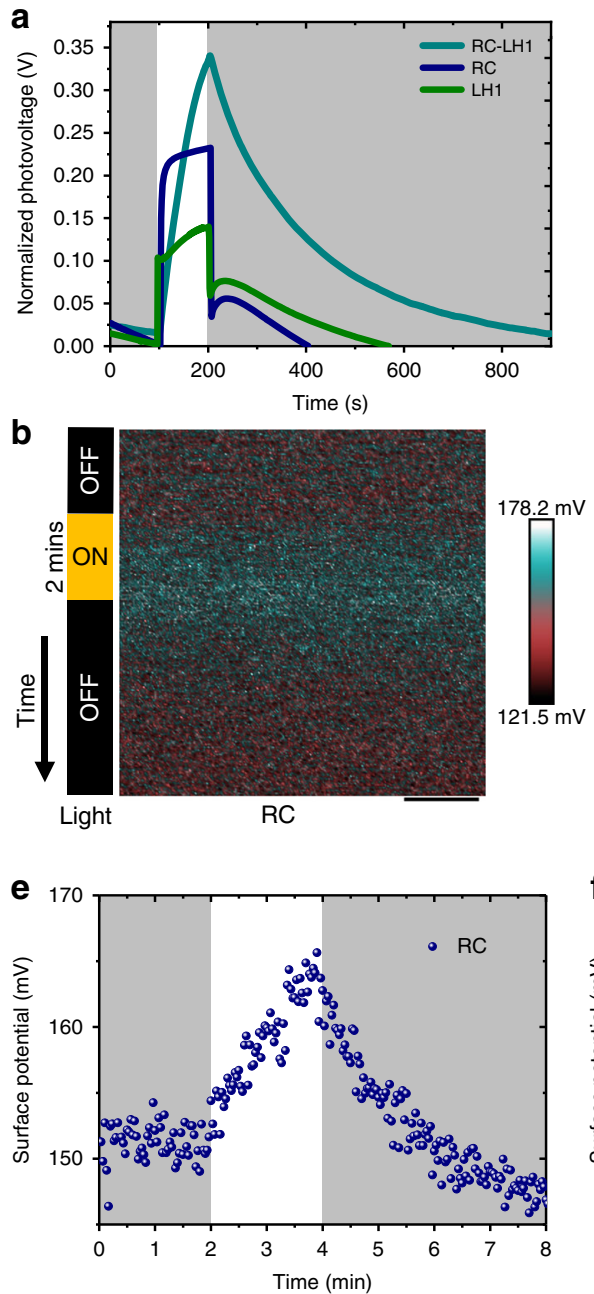

C

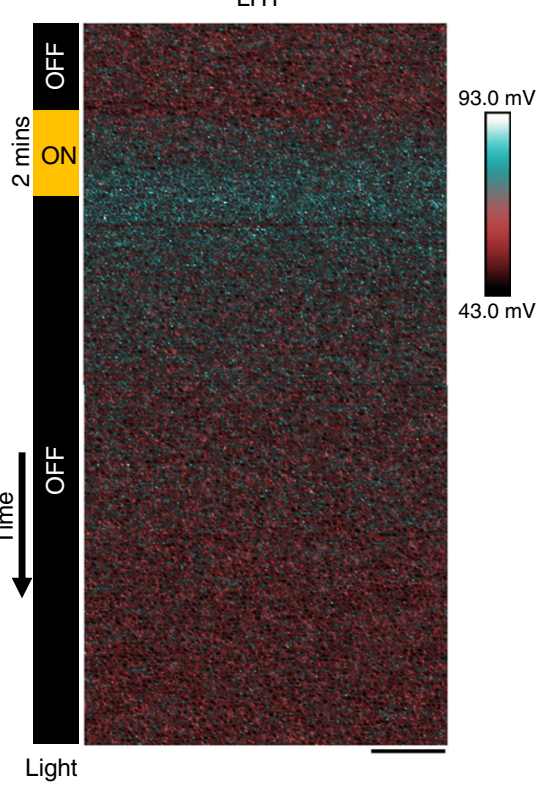

d

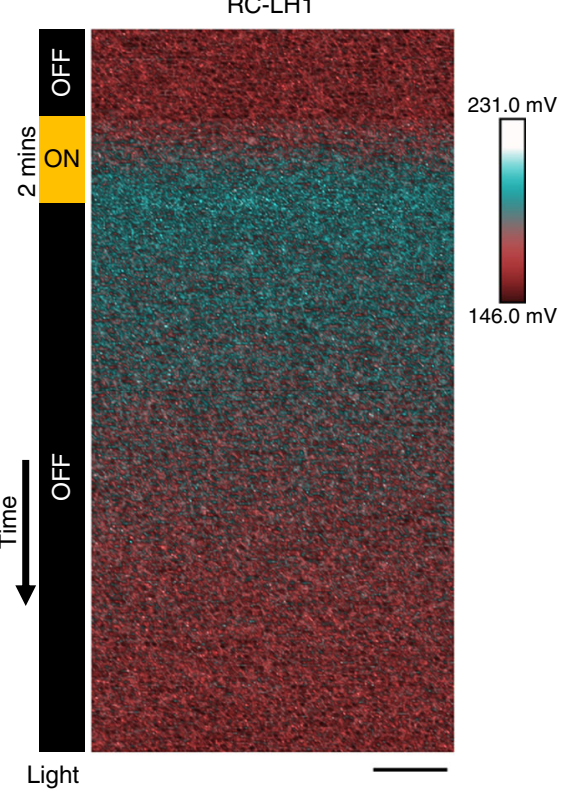

f

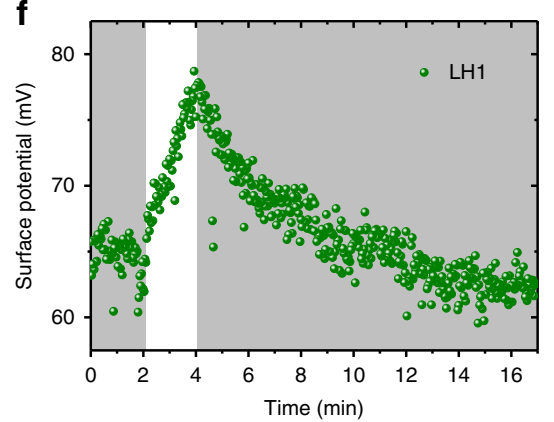

g



Fig. 4 Photocharging and dark discharging of photoprotein films. a Bulk charge-discharge characteristics of $500 \mu \mathrm{m}$ films of three different photoproteins namely, reaction centres (RC), light harvesting 1 complexes (LH1) and reaction centre-light harvesting 1 complexes (RC-LH1), exposed to 100 s illumination. b-d KPFM (Kelvin-probe force microscopy) surface potential maps of $\mathbf{b}$ RC, $\mathbf{c}$ LH1, and $\mathbf{d}$ RC-LH1 films before, during and after a 2 min period of illumination; scale bars denote $200 \mathrm{~nm}$. e-g Changes in surface potential before, during and after a 2 min period of illumination of e RC, $\mathbf{f} \mathrm{LH} 1$, and $\mathbf{g}$ RC-LH1 films

insulating layer that enables the build-up of charge at the electrode/protein interfaces.

A factor in the use of RC-LH1 complexes as a material for charge storage is that it is known from experiments employing conductive atomic force microscopy that individual complexes conduct electrical current under an applied bias ${ }^{50-52}$. Such experiments typically interrogate a monolayer of protein oriented on a conductive surface, and it has been suggested that electron tunneling across an LH complex is facilitated by the carotenoid cofactors ${ }^{50-52}$. However, multilayers of these proteins, arranged randomly with respect to an applied bias, would not be expected to conduct electrical current over a long range. Given this, a possible reason that the photovoltage supported by an RC-LH1 multilayer increased as its thickness increased up to $500 \mu \mathrm{m}$ (Fig. 2b) is that the effective resistance of the multilayer also increased, enabling the build-up of a larger potential difference. Such an explanation seems more plausible than positing that thicker layers produced higher voltages because they were more absorbing, as photochemical activity leading to trapped charges would be expected to be confined to a minority of the structure comprising (probably several) protein layers close to either electrode, rather than the entire thickness of the multilayer. The decrease in photovoltage achieved by films thicker than $500 \mu \mathrm{m}$ (Fig. 2b) is attributed to poor light penetration to the $\mathrm{n}$-Si back electrode and its adjacent RCLH1 proteins that lessens the accumulation of negative charge at this interface.

The Rba. sphaeroides RC-LH1 photosynthetic complex is modular (Fig. 1b-d), and the RC and LH1 proteins can be purified as separate, functional entities (absorbance spectra shown in Supplementary Fig. 1). BPCs formed from isolated RCs or LH1 complexes also generated a photovoltage (Fig. 4a). On comparing charge-discharge characteristics for $500 \mu \mathrm{m}$ protein films, it was found the photovoltage reached at the end of a standard $200 \mathrm{~s}$ illumination was the highest for the combined RC-LH1 complex and lowest for the LH1 protein (Fig. 4a). The light harvesting capacity of isolated RCs is limited to its six bacteriochlorins and one carotenoid (Fig. 1c), but each RC-LH1 complex has an additional $32 \mathrm{BChl}$ and 32 carotenoid lightharvesting pigments (Fig. 1b, d). The lower photovoltage achieved by a film of RCs is therefore likely attributable to a strongly diminished light harvesting capacity even though, as they are smaller than an RC-LH1 complex, the concentration of RCs in a packed multilayer would be expected to be greater. Although the 



Fig. 5 Capacitances of the biophotonic power cells. a Galvanostatic charge-discharge over a 0-1 V range for biophotonic power cells (BPCs) with three different photoproteins namely, reaction centres (RC), light harvesting 1 complexes (LH1), and reaction centre-light harvesting 1 complexes (RC-LH1), at an applied current density of $2 \mu \mathrm{A} \mathrm{cm}{ }^{-2}$. b Galvanostatic charge-discharge curves for an LH1 BPC at different current densities. c Cyclic voltammetry curves for the three BPCs. d Specific capacitance of the three BPCs; capacitance presented is the average of at least three measurements and the error bar denotes standard deviation. e Specific capacitance of an LH1 BPC at different applied current densities. $\mathbf{f}$ Capacitance gradient $V_{(\mathrm{dC} / \mathrm{dZ})}$ as a function of drive voltage for the three types of cell

pigment content of the isolated LH1 antenna protein is closer to that of RC-LH1 complexes, the absence of the RC means that light harvesting is not translated into a meta-stable charge separation, but rather energy is lost as emission and heat. Nevertheless, a photovoltage is still obtained because LH1 is capable of acting in a manner akin to an organic semiconductor, injecting excited electrons into the FTO electrode and becoming positively charged, or receiving electrons from the n-Si electrode and becoming negatively charged (Supplementary Fig. 2). However, the number of photo-accumulated charges is likely lower than in RC or RC-LH1 complexes because the lifetime of the LH1 excited state (estimated as 680 ps at room temperature ${ }^{53}$ ) is very short relative to the $1-5 \mathrm{~s}$ lifetime of the charge-separated state formed in $\mathrm{RC}$ and $\mathrm{RC}-\mathrm{LH} 1$ complexes.
Kelvin-probe microscopy to assess potential build-up and decay. Charging and discharging of the different types of BPC were further investigated by recording the surface potentials of the three types of protein film by KPFM. Films coated on an FTO substrate were scanned using a conductive $\mathrm{Pt} / \mathrm{Ir}$ probe before, during and after illumination. For all three films, the surface potential measured along a $1000 \mathrm{~nm}$ trajectory increased after light-on and decayed gradually after light-off (Fig. 4b-d), confirming the generation of a photovoltage. In good accord with the trend in overall device photovoltage (Fig. 4a), the averaged surface potential shift (Fig. 4e-g) was highest for the RC-LH1 films $(\sim 30 \mathrm{mV})$ and lower for LH1 or RC films $(\sim 15 \mathrm{mV})$. The time for relaxation of the surface potential shift was also longest for RCLH1 films $(\sim 15 \mathrm{~min})$ and shortest for RC films ( 3 min) 


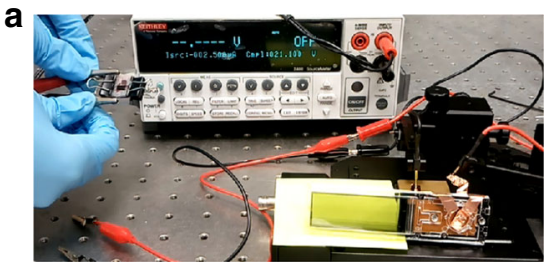

Cell connected to source meter

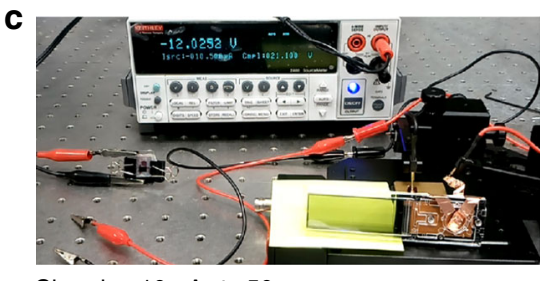

Charging $10 \mu \mathrm{A}, t=50 \mathrm{~s}$



Charging $10 \mu \mathrm{A}, t=0 \mathrm{~s}$



Cell connected to LED display

Fig. 6 Storage of externally injected charges by a single biophotonic power cell. a A single-biophotonic power cell (BPC) with RC-LH1 (reaction centre-light harvesting 1 complexes) was connected to source meter. $\mathbf{b}$ Charge was injected into the cell by applying a constant current of $10 \mu \mathrm{A}$. $\mathbf{c}$ The cell was charged for $50 \mathrm{~s}$. d The charged cell powered an LED display for approximately $1 \mathrm{~s}$

(Fig. $4 \mathrm{e}-\mathrm{g}$ ). These relaxation times of minutes confirmed that the potential shifts were due to the photogeneration of trapped charges rather than photochemistry within individual proteins, where relaxation occurs in a few seconds.

The KPFM and macroscopic photovoltage measurements indicated that all three proteins can generate and store charge. However, the extent of charge accumulation by each protein under these conditions was determined by its light-harvesting capacity and photochemical activity rather than its innate charge storage capacity. As a result, the discharge times in Figs. 2 and 4 did not necessarily convey the relative charge storage capacities of the three proteins as each was charged to different extent under illumination. To investigate the capacitance of each of the proteins at a macroscopic level, galvanostatic charge-discharge measurements and $\mathrm{CV}$ were performed on the two electrode devices (Fig. 5a-f). Near-symmetrical charge-discharge responses were obtained over a $0-1 \mathrm{~V}$ voltage window at an applied current of $2 \mu \mathrm{A} \mathrm{cm}^{-2}$, indicating a good capacitive behavior (Fig. $5 \mathrm{a}$ ). The LH1 photoprotein exhibited the largest area under the curve, indicating the highest capacitance, followed by RC-LH1 and then RC. Galvanostatic charge-discharge responses of the LH1 cell over a range of applied currents from 2 to $12 \mu \mathrm{A} \mathrm{cm}^{-2}$ were also near-symmetrical, confirming the capacitive behavior (Fig. 5b).

Microscale and macroscale capacitance measurements. CV confirmed the higher capacitance of the LH1 cells compared to the RC-LH1 and RC cells, the area of the near-rectangular CV plot being the highest for LH1 (Fig. 5c, and see Supplementary Fig. 3). It is known that the capacitance is proportional to the area under the CV curve ( $i v$-where $i$ and $v$ are the current and applied potential, respectively). Using the galvanostatic charge-discharge responses, the specific capacitance can be determined from the applied current, the active area and the slope of the discharge curve. From the charge-discharge profiles the specific capacitance was found to be the highest for LH1 films $\left(\approx 0.19 \mathrm{~F} \mathrm{~m}^{-2}\right)$, followed by RC-LH1 films $\left(\approx 0.14 \mathrm{~F} \mathrm{~m}^{-2}\right)$ and RC films $\left(\approx 0.11 \mathrm{~F} \mathrm{~m}^{-2}\right)$ (Fig. $\left.5 \mathrm{~d}\right)$. Over a $2-12 \mu \mathrm{A} \mathrm{cm}^{-2}$ range of applied current, the LH1 cells exhibited a maximal capacitance at $2 \mu \mathrm{A} \mathrm{cm}^{-2}$ and the capacitance did not drop beyond $0.12 \mathrm{~F} \mathrm{~m}^{-2}$ over the entire range (Fig. 5e).

These differences in capacitance were confirmed on the microscopic level using SCM measurements in which the same amount of injected charge was made available to each type of protein film by applying a range of drive voltages from an external source. The determined gradient of capacitance between the AFM tip and the sample, $V_{(\mathrm{dC} / \mathrm{dz})}$, is an indirect measure of the dielectric constant of the sample. SCM maps were recorded over a defined area of RC-LH1, LH1, or RC film at six different drive voltages and converted into average values of $V_{(\mathrm{dC} / \mathrm{dz})}$ to establish uniformity (Supplementary Fig. 4). A plot of the capacitance gradient averaged across the scanned film area as a function of drive voltage (Fig. 5f) showed that $V_{(\mathrm{dC} / \mathrm{dZ})}$ was highest for LH1 films and lowest for RC films at each drive voltage, in agreement with the trend in specific capacitances derived from charge-discharge and CV measurements (see above). Despite these measured capacitances exhibiting the order LH1 $>$ RC-LH1 > RC, the observed photovoltage displayed the trend RC-LH1 > RC > LH1, consistent with the RC-LH1 complex being superior to RCs in light harvesting and superior to LH1 complexes in the translation of short-lived excited states into long-lived charge-separated states.

Proof-of-concept demonstration and outlook. Finally, the utility of the $500 \mu \mathrm{m}$ RC-LH1 BPCs was demonstrated through their ability to power a low-consumption light emitting diode (LED) display. In the demonstration depicted in Fig. 6, a single RC-LH1 cell was charged by a constant current of $10 \mu \mathrm{A}$ for $50 \mathrm{~s}$ (Fig. 6a-c). This provided sufficient charge to power an LED display for approximately $1 \mathrm{~s}$ (Fig. 6d). In a second experiment of this type (Supplementary Fig. 5) three RC-LH1 cells connected in series were charged by a $1 \mathrm{~mA}$ current for $2 \mathrm{~s}$ and were able to power an LED display for up to $5 \mathrm{~s}$. Photo-charging of a bank of four RC-LH1 cells at 1 sun illumination for 5 min powered an LED display for about a second (Supplementary Fig. 6). The mode of operation of the BPCs in these charging demonstrations can be best understood in terms of a parallelplate capacitor in which the BPC plays a dual role of capacitor and, when illuminated, an integrated power source (Supplementary Fig. 7)

\section{Discussion}

In summary, we show with microscopic and macroscopic evidences that the purple bacterial photoprotein multilayers exhibit prolonged charge storage. The phenomenon unveiled not only 
casts new light on the photonic and electronic properties of the photosynthetic proteins but also presents a promising application in energy research. Looking to future developments, a key feature of the BPCs described in this report was the use of protein multilayers to generate the photovoltage and store charges. As these films were fabricated by simply drop-casting a concentrated solution of detergent-solubilized protein into a pre-formed well it is to be expected that individual proteins had a random orientation within the multilayer. One way to possibly boost the photovoltages obtained may be to control the orientation of individual RC-LH1 proteins throughout the multilayer such that there is alignment of the dipoles created between the $\mathrm{P}$ and $\mathrm{Q}_{\mathrm{B}}$ termini of the RC on photoexcitation. Large photovoltages obtained from micron thick crystals of the Photosystem I (PSI) $\mathrm{RC}$ from Pisum sivatum (pea) in response to strong $\left(1.1 \mathrm{~W} \mathrm{~cm}^{-2}\right)$ $660 \mathrm{~nm}$ laser excitation have been attributed to a superposition of photoinduced dipoles across uniformly oriented PSI proteins within the crystal lattice ${ }^{54,55}$. In the present case, imposing a uniform alignment on the RC-LH1 complexes within a film could both maximize desired redox interactions at each electrode surface (i.e., $\mathrm{Q}_{\mathrm{B}}$ oxidation at the $\mathrm{FTO}$-glass anode or $\mathrm{P}^{+}$reduction at the $\mathrm{n}$-Si cathode) and align the dipoles created across individual RC-LH1 complexes, both of which could potentially lead to a higher photovoltage. However, it is worth noting that achieving such control over protein orientation in a thick, multilayer film is challenging, and an attractive feature of the unoriented protein films used in the present work is their simplicity of fabrication.

\section{Methods}

Biological materials. Integrated power cells were prepared using either the PufXdeficient RC-LH1 complex from the purple photosynthetic bacterium $R b a$. sphaeroides or the component RC or LH1 proteins. These were isolated from three strains of Rba. sphaeroides in which either RC-LH1, RC, or LH1 was the sole pigment-protein in the cell ${ }^{30,31,56}$. Bacterial cells were grown in M22+ medium under dark/semiaerobic conditions ${ }^{57}$. His-tagged RCs were solubilized from photosynthetic membranes using $1.5 \%(\mathrm{w} / \mathrm{v}) \mathrm{n}$-dodecyl-N,N-dimethylamine-N-oxide (LDAO) and purified by nickel affinity chromatography on a FF Ni-NTA column (GE Healthcare), followed by size exclusion chromatography on a Superdex 200 16/60 column (GE Heathcare) ${ }^{56}$. RCs were exchanged into $20 \mathrm{mM}$ Tris ( $\mathrm{pH}$ 8) $/ 0.04 \% \mathrm{n}$-dodecyl $\beta$-D-maltopyranoside (DDM) during size exclusion chromatography and concentrated in this buffer to an absorbance at $802 \mathrm{~nm}\left(\mathrm{~A}^{802}\right)$ of $\approx 350$ absorbance units. RC-LH1 complexes which had a His-tag on the RC component were isolated from photosynthetic membranes using $2 \%(w / v)(D D M)$ and also purified using nickel affinity chromatography on a FF Ni-NTA column (GE Healthcare) followed by size exclusion chromatography on a Superdex 200 16/ 60 column (GE Heathcare) ${ }^{58}$. RC-LH1 complexes were concentrated in $20 \mathrm{mM}$ Tris $(\mathrm{pH} 8) / 0.04 \% \mathrm{DDM}$ to an $\mathrm{A}^{875}$ of $\approx 600$ absorbance units. For LH1 complexes, cells from $3 \mathrm{~L}$ of culture medium were harvested by centrifugation and resuspended in $100 \mathrm{~mL}$ of $20 \mathrm{mM}$ Tris ( $\mathrm{pH} \mathrm{8}$ ), containing several crystals of DNAseI. Cells were lysed at 20,000 psi in a Constant Systems cell disruptor and cell debris was removed by centrifugation at $27,000 \times g$ for $15 \mathrm{~min}$ at $4{ }^{\circ} \mathrm{C}$. The supernatant was loaded on $15 / 40 \%(\mathrm{w} / \mathrm{v})$ sucrose step gradients made up in $20 \mathrm{mM}$ Tris ( $\mathrm{pH} 8.0$ ), and the gradients were ultracentrifuged at $113,000 \times g$ and $4{ }^{\circ} \mathrm{C}$ for $2 \mathrm{~h}$. Membranes were harvested from the interface, diluted with $20 \mathrm{mM}$ Tris $(\mathrm{pH} \mathrm{8.0)}$ and pelleted by ultracentrifugation at $113,000 \times g$ and $4{ }^{\circ} \mathrm{C}$ for $2 \mathrm{~h}$. The membrane pellet was then resuspended overnight in $20 \mathrm{mM}$ Tris ( $\mathrm{pH} 8.0$ ) to an optical density at $875 \mathrm{~nm}$ of approximately $40 \mathrm{~cm}^{-1}$. LH1 was isolated from membranes by the addition of $3.0 \%$ octyl glucoside (OG) followed by stirred incubation in the dark at room temperature for 90 mins. Membrane debris was removed by centrifugation at $113,000 \times g$ for $30 \mathrm{~min}$ at $4{ }^{\circ} \mathrm{C}$. The supernatant was passed through $3 \times 5 \mathrm{~mL}$ DEAE anion exchange columns (GE Heathcare) that had been equilibrated with $75 \mathrm{~mL}$ of $20 \mathrm{mM}$ Tris (pH 8.0)/0.1\% OG. The columns were washed with $150 \mathrm{~mL}$ of $20 \mathrm{mM}$ Tris $(\mathrm{pH} 8.0) / 0.1 \% \mathrm{OG} / 150 \mathrm{mM} \mathrm{NaCl}$ and bound protein eluted with $50 \mathrm{~mL}$ of $20 \mathrm{mM}$ Tris ( $\mathrm{pH} 8.0) / 0.1 \%$ OG/300 mM NaCl. Eluted protein was further purified using a Superdex $20016 / 60$ column (GE Heathcare) pre-equilibrated in, and run with, $20 \mathrm{mM}$ Tris ( $\mathrm{pH}$ 8)/0.04\% DDM to achieve detergent exchange from OG to DDM. Fractions with a ratio of absorbance at $875 \mathrm{~nm}$ and $280 \mathrm{~nm}$ $\left(\mathrm{A}^{875} / \mathrm{A}^{280}\right)$ above 2.0 were pooled and concentrated using a $100 \mathrm{kDa}$ cut-off spin concentrator (Amicon) until the $\mathrm{A}^{875}$ was at least $600 \mathrm{~cm}^{-1}$. All purified proteins were aliquoted and stored at $-80^{\circ} \mathrm{C}$ until use.

Experimental design and device construction. Aliquots $(20-1000 \mu \mathrm{L})$ of concentrated protein solution were drop-cast and vacuum dried onto FTO-glass in a Parafilm well that had an area of $0.05 \pm 0.005 \mathrm{~cm}^{2}$ and a uniform depth varying between 0.1 and $2 \mathrm{~mm}$. Once the protein layer was dried a precleaned $\mathrm{n}$-Si counter electrode was placed on the well and held in place using binder clips.

Device-photovoltage measurements. All photovoltage measurements were carried out using a Keithley K2400 source meter under white light excitation at an intensity of $100 \mathrm{~mW} \mathrm{~cm}^{-2}$ approximating to 1 sun illumination.

Microscopic studies on charge build-up and decay. KPFM was carried out using a Dimension ICON microscope (Bruker Nano Surface, Santa Barbara, CA). Amplitude modulation was used to obtain a high signal to noise ratio, and all measurements were performed in a dual pass mode to eliminate any topography effects. Scans were carried out using Pt/Ir coated SCM-PIT probes with an applied bias and the surface potential of the protein film determined from the contact potential difference (CPD) between the tip and the film. As the probe traversed the sample scan area the film was illuminated for 2 min using a tungsten-halogen lamp at an incident light intensity of $10 \mathrm{~mW} \mathrm{~cm}^{-2}$ and surface scanning was continued during the subsequent dark period until the base surface potential was reached. For scans in which the base potential did not reach the original level after illumination was turned off within the scan range of $0-1000 \mathrm{~nm}$, the scan was instantly continued in the same scan area (from the 1000 to $0 \mathrm{~nm}$ position) until the original base potential was reached. This produced scan maps for the RC-LH1 and LH1 films that were twice the area of those for the RC. For SCM the gradient of capacitance between the probe and the sample, $V_{(\mathrm{dC} / \mathrm{dZ})}$, was measured to give an indirect measure of the capacitance of the protein film. All KPFM and SCM measurements were performed on $50 \mu \mathrm{m}$ thick protein films drop cast onto FTO glass. Thicker protein films were not used because they had a high surface roughness that resulted in high noise in the potential/capacitance scans. The illumination intensity $10 \mathrm{~mW} \mathrm{~cm}^{-2}$ was the maximum achievable using a bespoke illumination setup.

Device-capacitance measurements. CV and galvanostatic charge/discharge measurements were conducted using Solartron System 1470E. Capacitance was quantified from the charge-discharge patterns and the estimated capacitance could be attributed mainly to the proteins, neglecting the capacitance resulting from the surrounding paraffin film. The paraffin film, which had a dielectric constant in the range of 1.9-2.5, resulted in a negligibly low capacitance in the range of a few $\mathrm{pF}$ which was 5 orders of magnitude lower than the overall device capacitance.

\section{Data availability}

All relevant data will be made available upon reasonable request. Requests for data should be addressed to S.C.T.

Received: 10 October 2018 Accepted: 25 January 2019 Published online: 22 February 2019

\section{References}

1. Zazubovich, V. \& Jankowiak, R. Biophotonics of photosynthesis. Photonics, Volume 4: Biomedical Photonics, Spectroscopy, and Microscopy, 129 (Hoboken, New Jersey, John Wiley \& Sons, Inc., 2015).

2. Scholes, G. D., Fleming, G. R., Olaya-Castro, A. \& van Grondelle, R. Lessons from nature about solar light harvesting. Nat. Chem. 3, 763-774 (2011).

3. Croce, R. \& van Amerongen, H. Natural strategies for photosynthetic light harvesting. Nat. Chem. Biol. 10, 492-501 (2014).

4. Mirkovic, T., Ostroumov, E. E., Anna, J. M., van Grondelle, R. \& Scholes, G. D. Light absorption and energy transfer in the antenna complexes of photosynthetic organisms. Chem. Rev. 117, 249-293 (2016).

5. Xue, X. et al. Flexible self-charging power cell for one-step energy conversion and storage. Adv. Energy Mater. 4, 1301329 (2014).

6. Chen, T. et al. An integrated "energy wire" for both photoelectric conversion and energy storage. Angew. Chem. Int. Ed. 51, 11977-11980 (2012).

7. Wang, Z. L. \& Wu, W. Nanotechnology-enabled energy harvesting for self-powered micro-/nanosystems. Angew. Chem. Int. Ed. 51, 11700-11721 (2012).

8. Ramadoss, A. et al. Piezoelectric-driven self-charging supercapacitor power cell. ACS Nano 9, 4337-4345 (2015).

9. Kathy, L. Materials in Energy Conversion, Harvesting, and Storage. (Hoboken, New Jersey, John Wiley \& Sons, Inc., 2014).

10. Xue, X., Wang, S., Guo, W., Zhang, Y. \& Wang, Z. L. Hybridizing energy conversion and storage in a mechanical-to-electrochemical process for selfcharging power cell. Nano Lett. 12, 5048-5054 (2012).

11. Kim, Y.-S. et al. Highly porous piezoelectric PVDF membrane as effective lithium ion transfer channels for enhanced self-charging power cell. Nano Energy 14, 77-86 (2015). 
12. Niu, S., Wang, X., Yi, F., Zhou, Y. S. \& Wang, Z. L. A universal self-charging system driven by random biomechanical energy for sustainable operation of mobile electronics. Nat. Commun. 6, 8975 (2015).

13. Qi, Y. \& McAlpine, M. C. Nanotechnology-enabled flexible and biocompatible energy harvesting. Energy Environ. Sci. 3, 1275-1285 (2010).

14. Xu, S., Hansen, B. J. \& Wang, Z. L. Piezoelectric-nanowire-enabled power source for driving wireless microelectronics. Nat. Commun. 1, 93 (2010).

15. Wu, F., Cai, W., Yeh, Y.-W., Xu, S. \& Yao, N. Energy scavenging based on a single-crystal PMN-PT nanobelt. Sci. Rep. 6, 22513 (2016).

16. Zhu, G., Chen, J., Zhang, T., Jing, Q. \& Wang, Z. L. Radial-arrayed rotary electrification for high performance triboelectric generator. Nat. Commun. 5, 3426 (2014).

17. Chun, J. et al. Boosted output performance of triboelectric nanogenerator via electric double layer effect. Nat. Commun. 7, 12985 (2016).

18. Zi, Y. et al. Effective energy storage from a triboelectric nanogenerator. Nat. Commun. 7, 10987 (2016)

19. Skunik-Nuckowska, M. et al. Integration of solid-state dye-sensitized solar cell with metal oxide charge storage material into photoelectrochemical capacitor. J. Power Sources 234, 91-99 (2013).

20. Chen, H.-W. et al. Plastic dye-sensitized photo-supercapacitor using electrophoretic deposition and compression methods. J. Power Sources 195 , 6225-6231 (2010).

21. Xu, J., Chen, Y. \& Dai, L. Efficiently photo-charging lithium-ion battery by perovskite solar cell. Nat. Commun. 6, 8103 (2015).

22. Liao, S. et al. Integrating a dual-silicon photoelectrochemical cell into a redox flow battery for unassisted photocharging. Nat. Commun. 7, 11474 (2016).

23. Niwa, S. et al. Structure of the LH1-RC complex from thermochromatium tepidum at $3.0 \AA$ A . Nature 508, 228-232 (2014).

24. Qian, P. et al. Three-dimensional structure of the Rhodobacter sphaeroides RC-LH1-PufX complex: dimerization and quinone channels promoted by PufX. Biochemistry 52, 7575-7585 (2013).

25. Jones, M. R. The petite purple photosynthetic powerpack. Biochem. Soc. Trans. 37, 400-407 (2009).

26. Zinth, W. \& Wachtveitl, J. The first picoseconds in bacterial photosynthesisultrafast electron transfer for the efficient conversion of light energy. Chemphyschem 6, 871-880 (2005).

27. Ravi, S. K. \& Tan, S. C. Progress and perspectives in exploiting photosynthetic biomolecules for solar energy harnessing. Energy Environ. Sci. 8, 2551-2573 (2015).

28. Ravi, S. K., Udayagiri, V. S., Suresh, L. \& Tan, S. C. Emerging role of the bandstructure approach in biohybrid photovoltaics: a path beyond bioelectrochemistry. Adv. Funct. Mater. 28, 1705305 (2018).

29. Ravi, S. K. et al. Photosynthetic bioelectronic sensors for touch perception, $\mathrm{UV}$-detection, and nanopower generation: toward self-powered E-skins. $A d v$. Mater. 30, 1802290 (2018).

30. Ravi, S. K. et al. A mechanoresponsive phase-changing electrolyte enables fabrication of high-output solid-state photobioelectrochemical devices from pigment-protein multilayers. Adv. Mater. 30, 1704073 (2018).

31. Ravi, S. K. et al. Enhanced output from biohybrid photoelectrochemical transparent tandem cells integrating photosynthetic proteins genetically modified for expanded solar energy harvesting. Adv. Energy Mater. 7, 1601821 (2017).

32. Zhao, F. et al. Light-induced formation of partially reduced oxygen species limits the lifetime of photosystem 1-based biocathodes. Nat. Commun. 9, 1973 (2018).

33. Wenzel, T., Härtter, D., Bombelli, P., Howe, C. J. \& Steiner, U. Porous translucent electrodes enhance current generation from photosynthetic biofilms. Nat. Commun. 9, 1299 (2018).

34. Saper, G. et al. Live cyanobacteria produce photocurrent and hydrogen using both the respiratory and photosynthetic systems. Nat. Commun. 9, 2168 (2018).

35. Kornienko, N., Zhang, J. Z., Sakimoto, K. K., Yang, P. \& Reisner, E. Interfacing nature's catalytic machinery with synthetic materials for semi-artificial photosynthesis. Nat. Nanotechnol. 13, 890 (2018).

36. Siebert, C. A. et al. Molecular architecture of photosynthetic membranes in Rhodobacter sphaeroides: the role of PufX. EMBO J. 23, 690-700 (2004).

37. Walz, T., Jamieson, S. J., Bowers, C. M., Bullough, P. A. \& Hunter, C. N. Projection structures of three photosynthetic complexes from Rhodobacter sphaeroides: LH2 at $6 \AA$, LH1 and RC-LH1 at $25 \AA$. J. Mol. Biol. 282, 833-845 (1998).

38. Dezi, M. et al. Stabilization of charge separation and cardiolipin confinement in antenna-reaction center complexes purified from Rhodobacter sphaeroides. Biochim. Biophys. Acta 1767, 1041-1056 (2007).

39. Comayras, F., Jungas, C. \& Lavergne, J. Functional consequences of the organization of the photosynthetic apparatus in Rhodobacter sphaeroides, I. Quinone domains and excitation transfer in chromatophores and reaction center antenna complexes. J. Biol. Chem. 280, 11203-11213 (2005).
40. Francia, F. et al. Light-harvesting complex 1 stabilizes $\mathrm{P}^{+} \mathrm{Q}_{\mathrm{B}}{ }^{-}$charge separation in reaction centers of Rhodobacter sphaeroides. Biochemistry 43 , 14199-14210 (2004).

41. Kropacheva, T. N. \& Hoff, A. J. Electrochemical oxidation of bacteriochlorophyll a in reaction centers and antenna complexes of photosynthetic bacteria. J. Phys. Chem. B 105, 5536-5545 (2001).

42. Robert, B., Lutz, M. \& Tiede, D. M. Selective photochemical reduction of either of the two bacteriopheophytins in reaction centers of Rps. sphaeroides R-26. FEBS Lett. 183, 326-330 (1985).

43. Mar, T., Picorel, R. \& Gingras, G. Phototrapping of doubly reduced monomeric bacteriochlorophyll in the photoreaction center of Ectothiorhodospira sp. Biochemistry 32, 1466-1470 (1993).

44. Blaise, G. Charge localization and transport in disordered dielectric materials. J. Electrost. 50, 69-89 (2001).

45. Brédas, J.-L., Sargent, E. H. \& Scholes, G. D. Photovoltaic concepts inspired by coherence effects in photosynthetic systems. Nat. Mater. 16, 35 (2017).

46. Warren, J. J., Winkler, J. R. \& Gray, H. B. Hopping maps for photosynthetic reaction centers. Coord. Chem. Rev. 257, 165-170 (2013).

47. Takshi, A., Madden, J. D. \& Beatty, J. T. Diffusion model for charge transfer from a photosynthetic reaction center to an electrode in a photovoltaic device. Electrochim. Acta 54, 3806-3811 (2009).

48. Singh, V. K. et al. Biohybrid photoprotein-semiconductor cells with deep-lying redox shuttles achieve a $0.7 \mathrm{~V}$ photovoltage. Adv. Funct. Mater. 28, 1703689 (2017).

49. Trammell, S. A., Spano, A., Price, R. \& Lebedev, N. Effect of protein orientation on electron transfer between photosynthetic reaction centers and carbon electrodes. Biosens. Bioelectron. 21, 1023-1028 (2006).

50. Sumino, A., Dewa, T., Sasaki, N., Kondo, M. \& Nango, M. Electron conduction and photocurrent generation of a light-harvesting/reaction center core complex in lipid membrane environments. J. Phys. Chem. Lett. 4, 1087-1092 (2013).

51. Kondo, M. et al. Photocurrent and electronic activities of oriented-His-tagged photosynthetic light-harvesting/reaction center core complexes assembled onto a gold electrode. Biomacromolecules 13, 432-438 (2012).

52. Stamouli, A., Frenken, J., Oosterkamp, T., Cogdell, R. \& Aartsma, T. The electron conduction of photosynthetic protein complexes embedded in a membrane. FEBS Lett. 560, 109-114 (2004).

53. Monshouwer, R., Abrahamsson, M., Van Mourik, F. \& Van Grondelle, R. Superradiance and exciton delocalization in bacterial photosynthetic lightharvesting systems. J. Phys. Chem. B 101, 7241-7248 (1997).

54. Volotsenko, I., Molotskii, M., Borovikova, A., Nelson, N. \& Rosenwaks, Y. Evidence for deep acceptor centers in plant photosystem I crystals. J. Phys. Chem. B 119, 1374-1379 (2015).

55. Toporik, H. et al. Large photovoltages generated by plant photosystem I crystals. Adv. Mater. 24, 2988-2991 (2012).

56. Swainsbury, D. J., Friebe, V. M., Frese, R. N. \& Jones, M. R. Evaluation of a biohybrid photoelectrochemical cell employing the purple bacterial reaction centre as a biosensor for herbicides. Biosens. Bioelectron. 58, 172-178 (2014).

57. Jones, M. R., Heer-Dawson, M., Mattioli, T. A., Hunter, C. N. \& Robert, B. Site-specific mutagenesis of the reaction centre from Rhodobacter sphaeroides studied by Fourier transform Raman spectroscopy: mutations at tyrosine M210 do not affect the electronic structure of the primary donor. FEBS Lett. 339, 18-24 (1994).

58. Friebe, V. M. et al. Plasmon-enhanced photocurrent of photosynthetic pigment proteins on nanoporous silver. Adv. Funct. Mater. 26, 285-292 (2016).

\section{Acknowledgments}

S.K.R. thanks Dr. Kawsar Ali, University of Oxford, for fruitful technical discussion. S.C T. acknowledges the financial support from Ministry of Education Academic Research Fund (R-284-000-161-114 and R-284-000-174-114). M.R.J. acknowledges support from the Biotechnology and Biological Sciences Research Council (BBSRC-project BB/ I022570/1) and the BrisSynBio Synthetic Biology Research Centre at the University of Bristol (BB/L01386X/1) funded by the BBSRC and Engineering and Physical Sciences Research Council (EPSRC) of the UK.

\section{Author contributions}

S.K.R. and S.C.T. conceived the concept presented and designed the experiments. M.R.J provided the biological materials. S.K.R. observed the prolonged charge storage effect. S.K.R., P.R., and K.H. performed the photovoltage experiments. S.K.R. and A.M.E. performed the galvanostatic charge-discharge and cyclic voltammetry measurements. The experiments were, in part, performed in the laboratories of, and in collaboration with J.W. A.M.E. and J.W. contributed to the experimental design, data analysis, and manuscript revisions. W.S., S.K.R., and F.Z. performed the KPFM and SCM measurements. S.K.R., M.R.J., and S.C.T. analyzed and interpreted the data and wrote the manuscript. S.C.T. supervised the project. 


\section{Additional information}

Supplementary Information accompanies this paper at https://doi.org/10.1038/s41467019-08817-7.

Competing interests: The authors declare no competing interests.

Reprints and permission information is available online at http://npg.nature.com/ reprintsandpermissions/

Journal peer review information: Nature Communications thanks Arash Takshi and the other anonymous reviewers for their contribution to the peer review of this work.

Publisher's note: Springer Nature remains neutral with regard to jurisdictional claims in published maps and institutional affiliations. (c) Open Access This article is licensed under a Creative Commons Attribution 4.0 International License, which permits use, sharing, adaptation, distribution and reproduction in any medium or format, as long as you give appropriate credit to the original author(s) and the source, provide a link to the Creative Commons license, and indicate if changes were made. The images or other third party material in this article are included in the article's Creative Commons license, unless indicated otherwise in a credit line to the material. If material is not included in the article's Creative Commons license and your intended use is not permitted by statutory regulation or exceeds the permitted use, you will need to obtain permission directly from the copyright holder. To view a copy of this license, visit http://creativecommons.org/ licenses/by/4.0/.

(C) The Author(s) 2019 